\title{
A new iteration process for equilibrium, variational inequality, fixed point problems, and zeros of maximal monotone operators in a Banach space
}

\author{
Siwaporn Saewan ${ }^{1 *}$ and Poom Kumam²
}

\author{
"Correspondence: \\ si_wa_pon@hotmail.com; \\ poom.kum@kmutt.ac.th \\ ${ }^{1}$ Department of Mathematics and \\ Statistics, Faculty of Science, Thaksin \\ University (TSU), Pa Phayom, \\ Phatthalung, 93110, Thailand \\ ${ }^{2}$ Department of Mathematics, \\ Faculty of Science, King Mongkut's \\ University of Technology Thonburi, \\ Bang Mod, Bangkok, 10140, \\ Thailand
}

\begin{abstract}
In this article, a new iterative process is introduced to approximate a common element of a fixed point set, the solutions of equilibrium problems, the solution set of variational inequality problems, and the set of zeros of maximal monotone operators in a uniformly smooth and strictly convex Banach space by using a hybrid projection method. Also, we prove new strong convergence theorems for this proposed iterative precess in a Banach space.
\end{abstract}

MSC: $47 \mathrm{H} 05 ; 47 \mathrm{H} 09 ; 47 \mathrm{H} 10$

Keywords: maximal monotone mappings; strong convergence; total quasi- $\phi$-asymptotically nonexpansive mappings; hybrid scheme; equilibrium problem; variational inequality problems

\section{Introduction}

Let $E$ be a real Banach space, $E^{*}$ be the dual space of $E$. A set-valued mapping $A: D(A) \subset$ $E \rightarrow E^{*}$ with graph $G(A)=\left\{\left(x, x^{*}\right): x^{*} \in A x\right\}$, domain $D(A)=\{x \in E: A x \neq \emptyset\}$, and range $R(A)=\cup\{A x: x \in D(A)\} . A$ is said to be monotone if $\left\langle x-y, x^{*}-y^{*}\right\rangle \geq 0$ whenever $x^{*} \in A x$, $y^{\prime \prime} \in A y$. A monotone operator $A$ is said to be maximal monotone if its graph is not properly contained in the graph of any other monotone operator. Let $A \subset E \times E^{*}$ be a maximal monotone operator. We consider the problem for finding $x \in E$

$$
0 \in A x,
$$

a point $x \in E$ is called a zero point of $A$. Denote by $A^{-1} 0$ the set of all points $x \in E$ such that $0 \in A x$. We know that if $A$ is maximal monotone, then the solution set $A^{-1} 0=\{x \in$ $D(A): 0 \in T x\}$ is closed and convex. One popular algorithm for approximating a solution of this problem is called the proximal point algorithm which was first proposed by Martinet [1] and studied further by Rockafellar [2] in Hilbert spaces. Since the proximal point algorithm weakly converges in general which is the proximal point algorithm is defined by $x_{0} \in E$ and

$$
x_{n+1}=J_{r_{n}} x_{n}, \quad \text { for } n=0,1,2,3, \ldots,
$$

\section{Springer}

(0) 2013 Saewan and Kumam; licensee Springer. This is an Open Access article distributed under the terms of the Creative Commons Attribution License (http://creativecommons.org/licenses/by/2.0), which permits unrestricted use, distribution, and reproduction in any medium, provided the original work is properly cited. 
where $\left\{r_{n}\right\} \subset(0, \infty)$ and $J_{r_{n}}$ are the resolvent of $A$. Solovov and Svaitor [3] proposed a modified proximal point algorithm which converges strongly to a solution of the equation $A^{-1} 0$ by using the projection method. Many problems in nonlinear analysis and optimization can be formulated by the proximal point algorithm (see [4-9]).

Let $E$ be a real Banach space with dual $E^{*}$ and let $C$ be a nonempty closed and convex subset of $E$. Let $f: C \times C \rightarrow \mathbb{R}$ be a bifunction. The equilibrium problem is to find $x \in C$ such that

$$
f(x, y) \geq 0, \quad \forall y \in C .
$$

The equilibrium problem is very general in the sense that it includes, as special cases, optimization problems, variational inequality problems, min-max problems, saddle point problem, fixed point problem, Nash EP. In 2008, Takahashi and Zembayashi [10, 11] introduced iterative sequences for finding a common solution of an equilibrium problem and a fixed point problem.

A mapping $A: D(A) \subset E \rightarrow E^{*}$ is said to be $\alpha$-inverse-strongly monotone if there exists a constant $\alpha>0$ such that

$$
\langle x-y, A x-A y\rangle \geq \alpha\|A x-A y\|^{2}, \quad \forall x, y \in C .
$$

If $A$ is $\alpha$-inverse strongly monotone, then it is $\frac{1}{\alpha}$-Lipschitz continuous, i.e.,

$$
\|A x-A y\| \leq \frac{1}{\alpha}\|x-y\|, \quad \forall x, y \in C .
$$

Let $C$ be a nonempty closed and convex subset of a real Banach space $E$. Let $A$ be a monotone operator from $C$ into $E$. The variational inequality problem for an operator A is to find $\hat{z} \in C$ such that

$$
\langle y-\hat{z}, A \hat{z}\rangle \geq 0, \quad \forall y \in C .
$$

The set of solutions of (1.4) is denoted by $\operatorname{VI}(A, C)$.

Let $C$ be a nonempty closed and convex subset of $E$. A mapping $T$ from $C$ into itself is said to be nonexpansive if

$$
\|T x-T y\| \leq\|x-y\|, \quad \forall x, y \in C .
$$

$T$ is said to be total asymptotically nonexpansive if there exist nonnegative real sequences $v_{n}, \mu_{n}$ with $v_{n} \rightarrow 0, \mu_{n} \rightarrow 0$ as $n \rightarrow \infty$ and a strictly increasing continuous function $\varphi: \mathbb{R}^{+} \rightarrow \mathbb{R}^{+}$with $\varphi(0)=0$ such that

$$
\left\|T^{n} x-T^{n} y\right\| \leq\|x-y\|+\mu_{n} \psi(\|x-y\|)+v_{n}, \quad \forall x, y \in C, \forall n \geq 1 .
$$

A point $x \in C$ is a fixed point of $T$ provided $T x=x$. Denote by $F(T)$ the fixed point set of $T$; that is, $F(T)=\{x \in C: T x=x\}$. A point $p$ in $C$ is called an asymptotic fixed point of 
$T$ [12] if $C$ contains a sequence $\left\{x_{n}\right\}$ which converges weakly to $p$ such that $\lim _{n \rightarrow \infty} \| x_{n}-$ $T x_{n} \|=0$. The asymptotic fixed point set of $T$ is denoted by $\widehat{F}(T)$.

The value of $x^{*} \in E^{*}$ at $x \in E$ will be denoted by $\left\langle x, x^{*}\right\rangle$ or $x^{*}(x)$. For each $p>1$, the generalized duality mapping $J_{p}: E \rightarrow 2^{E^{*}}$ is defined by

$$
J_{p}(x)=\left\{x^{*} \in E^{*}:\left\langle x, x^{*}\right\rangle=\|x\|^{p},\left\|x^{*}\right\|=\|x\|^{p-1}\right\}
$$

for all $x \in E$. In particular, $J=J_{2}$ is called the normalized duality mapping. If $E$ is a Hilbert space, then $J=I$, where $I$ is the identity mapping. Consider the functional defined by

$$
\phi(x, y)=\|x\|^{2}-2\langle x, J y\rangle+\|y\|^{2} \quad \text { for } x, y \in E .
$$

If $E$ is a Hilbert space, then $\phi(y, x)=\|y-x\|^{2}$. It is obvious from the definition of $\phi$ that

$$
(\|y\|-\|x\|)^{2} \leq \phi(y, x) \leq(\|y\|+\|x\|)^{2}, \quad \forall x, y \in E .
$$

$T$ is said to be $\phi$-nonexpansive $[13,14]$ if

$$
\phi(T x, T y) \leq \phi(x, y), \quad \forall x, y \in C .
$$

$T$ is said to be quasi- $\phi$-nonexpansive $[13,14]$ if $F(T) \neq \emptyset$ and

$$
\phi(p, T x) \leq \phi(p, x), \quad \forall x \in C \text { and } p \in F(T) .
$$

$T$ is said to be asymptotically $\phi$-nonexpansive [14] if there exists a sequence $\left\{k_{n}\right\} \subset[0, \infty)$ with $k_{n} \rightarrow 1$ as $n \rightarrow \infty$ such that

$$
\phi\left(T^{n} x, T^{n} y\right) \leq k_{n} \phi(x, y), \quad \forall x, y \in C
$$

$T$ is said to be quasi- $\phi$-asymptotically nonexpansive [14] if $F(T) \neq \emptyset$ and there exists a sequence $\left\{k_{n}\right\} \subset[0, \infty)$ with $k_{n} \rightarrow 1$ as $n \rightarrow \infty$ such that

$$
\phi\left(p, T^{n} x\right) \leq k_{n} \phi(p, x), \quad \forall x \in C, p \in F(T), \forall n \geq 1 .
$$

$T$ is said to be total quasi- $\phi$-asymptotically nonexpansive if $F(T) \neq \emptyset$ and there exist nonnegative real sequences $v_{n}, \mu_{n}$ with $v_{n} \rightarrow 0, \mu_{n} \rightarrow 0$ as $n \rightarrow \infty$ and a strictly increasing continuous function $\varphi: \mathbb{R}^{+} \rightarrow \mathbb{R}^{+}$with $\varphi(0)=0$ such that

$$
\phi\left(p, T^{n} x\right) \leq \phi(p, x)+v_{n} \varphi(\phi(p, x))+\mu_{n}, \quad \forall n \geq 1, \forall x \in C, p \in F(T) .
$$

A mapping $T$ is said to be uniformly L-Lipschitz continuous, if there exists a constant $L>0$ such that

$$
\left\|T^{n} x-T^{n} y\right\| \leq L\|x-y\|, \quad \forall x, y \in C .
$$


$T$ is said to be closed if for any sequence $\left\{x_{n}\right\} \subset C$ such that $\lim _{n \rightarrow \infty} x_{n}=x_{0}$ and $\lim _{n \rightarrow \infty} T x_{n}=y_{0}, T x_{0}=y_{0}$.

Remark 1.1 Every quasi- $\phi$-nonexpansive mapping implies a quasi- $\phi$-asymptotically nonexpansive mapping and a quasi- $\phi$-asymptotically nonexpansive mapping implies a total quasi- $\phi$-asymptotically nonexpansive mapping, but the converse is not true.

On the other hand, Alber [15] introduced that the generalized projection $\Pi_{C}: E \rightarrow C$ is a map that assigns to an arbitrary point $x \in E$ the minimum point of the functional $\phi(x, y)$, that is, $\Pi_{C} x=\bar{x}$, where $\bar{x}$ is the solution of the minimization problem

$$
\phi(\bar{x}, x)=\inf _{y \in C} \phi(y, x)
$$

The existence and uniqueness of the operator $\Pi_{C}$ follow from the properties of the functional $\phi(x, y)$ and strict monotonicity of the mapping $J$. Let $\Pi_{C}$ be the generalized projection from a smooth strictly convex and reflexive Banach space $E$ onto a nonempty closed convex subset $C$ of $E$. Then $\Pi_{C}$ is a closed relatively quasi-nonexpansive mapping from $E$ onto $C$ with $F\left(\Pi_{C}\right)=C$.

Matsushita and Takahashi [16] proposed the following hybrid iteration method with a generalized projection for a relatively nonexpansive mapping $T$ in a Banach space $E$ :

$$
\left\{\begin{array}{l}
x_{0} \in C \quad \text { chosen arbitrarily, } \\
y_{n}=J^{-1}\left(\alpha_{n} J x_{n}+\left(1-\alpha_{n}\right) J T x_{n}\right), \\
C_{n}=\left\{z \in C: \phi\left(z, y_{n}\right) \leq \phi\left(z, x_{n}\right)\right\}, \\
Q_{n}=\left\{z \in C:\left\langle x_{n}-z, J x_{0}-J x_{n}\right\rangle \geq 0\right\}, \\
x_{n+1}=\Pi_{C_{n} \cap Q_{n}} x_{0} .
\end{array}\right.
$$

They proved that $\left\{x_{n}\right\}$ converges strongly to $\Pi_{F(T)} x_{0}$. Many authors studied the methods for approximating fixed points of a countable family of (relatively quasi-) nonexpansive mappings (see [17-19]).

Recently, Qin et al. [20] considered a pair of asymptotically quasi- $\phi$-nonexpansive mappings. To be more precise, they proved the following results.

Theorem QCK Let E be a uniformly smooth and uniformly convex Banach space and C be a nonempty closed and convex subset of E. Let $T: C \rightarrow C$ be a closed and asymptotically quasi- $\phi$-nonexpansive mapping with the sequence $\left\{k_{n}^{(t)}\right\} \subset[1, \infty)$ such that $k_{n}^{(t)} \rightarrow 1$ as $n \rightarrow$ $\infty$ and $S: C \rightarrow C$ be a closed and asymptotically quasi- $\phi$-nonexpansive mapping with the sequence $\left\{k_{s}^{(t)}\right\} \subset[1, \infty)$ such that $k_{n}^{(s)} \rightarrow 1$ as $n \rightarrow \infty$. Let $\left\{\alpha_{n}\right\},\left\{\beta_{n}\right\},\left\{\gamma_{n}\right\}$, and $\left\{\delta_{n}\right\}$ be real number sequences in $[0,1]$. Assume that $T$ and $S$ are uniformly asymptotically regular on $C$ and $\Omega=F(T) \cap F(S)$ is nonempty and bounded. Let $\left\{x_{n}\right\}$ be a sequence generated in the 
following manner:

$$
\left\{\begin{array}{l}
x_{0} \in E \quad \text { chosen arbitrarily, } \\
C_{1}=C, \\
x_{1}=\Pi_{C_{1}} x_{0}, \\
z_{n}=J^{-1}\left(\beta_{n} J x_{n}+\gamma_{n} J\left(T^{n} x_{n}\right)+\delta_{n} J\left(S^{n} x_{n}\right)\right), \\
y_{n}=J^{-1}\left(\alpha_{n} J x_{n}+\left(1-\alpha_{n}\right) J z_{n}\right), \\
C_{n+1}=\left\{w \in C_{n}: \phi\left(w, y_{n}\right) \leq \phi\left(w, x_{n}\right)+\left(k_{n}-1\right) M_{n}\right\}, \\
x_{n+1}=\Pi_{C_{n+1}} x_{0},
\end{array}\right.
$$

where $k_{n}=\max \left\{k_{n}^{(t)}, k_{n}^{(s)}\right\}$ for each $n \geq 1, J$ is the duality mapping on $E, M_{n}=\sup \left\{\phi\left(z, x_{n}\right)\right.$ : $z \in \Omega\}$ for each $n \geq 1$. Assume that the control sequences $\left\{\alpha_{n}\right\},\left\{\beta_{n}\right\},\left\{\gamma_{n}\right\}$, and $\left\{\delta_{n}\right\}$ satisfy the following restrictions:

(a) $\beta_{n}+\gamma_{n}+\delta_{n}=1, \forall n \geq 1$;

(b) $\liminf _{n \rightarrow \infty} \gamma_{n} \delta_{n}, \lim _{n \rightarrow \infty} \beta_{n}=0$;

(c) $0 \leq \alpha_{n}<1$ and $\lim \sup _{n \rightarrow \infty} \alpha_{n}<1$.

In 2008, Alber et al. [21] proved the strong convergence theorems to approximate a fixed point of a total asymptotically nonexpansive mapping in a Hilbert space. In 2011, Chang et $a l$. $[22,23]$ proved the strong convergence theorems for finding the set of fixed points of a total quasi- $\phi$-asymptotically nonexpansive mapping in the framework of Banach spaces.

Motivated and inspired by the work mentioned above, in this paper, we introduce a new hybrid projection algorithm for a pair of total quasi- $\phi$-asymptotically nonexpansive mappings for finding a set of solutions of the equilibrium problem, a zero point of maximal monotone operators, and a set of solutions of the variation inequality in a uniformly smooth and strictly convex Banach space.

\section{Preliminaries}

In this article, we denote the strong convergence and weak convergence of a sequence $\left\{x_{n}\right\}$ by $x_{n} \rightarrow x$ and $x_{n} \rightarrow x$, respectively.

A Banach space $E$ with the norm $\|\cdot\|$ is called strictly convex if $\left\|\frac{x+y}{2}\right\|<1$ for all $x, y \in E$ with $\|x\|=\|y\|=1$ and $x \neq y$. Let $U=\{x \in E:\|x\|=1\}$ be the unit sphere of $E$. A Banach space $E$ is called smooth if the $\operatorname{limit}_{\lim } \rightarrow 0 \frac{\|x+t y\|-\|x\|}{t}$ exists for each $x, y \in U$. It is also called uniformly smooth if the limit exists uniformly for all $x, y \in U$. The modulus of convexity of $E$ is the function $\delta:[0,2] \rightarrow[0,1]$ defined by

$$
\delta(\varepsilon)=\inf \left\{1-\left\|\frac{x+y}{2}\right\|: x, y \in E,\|x\|=\|y\|=1,\|x-y\| \geq \varepsilon\right\} .
$$

A Banach space $E$ is uniformly convex if and only if $\delta(\varepsilon)>0$ for all $\varepsilon \in(0,2]$. Let $p$ be a fixed real number with $p \geq 2$. A Banach space $E$ is said to be $p$-uniformly convex if there exists a constant $c>0$ such that $\delta(\varepsilon) \geq c \varepsilon^{p}$ for all $\varepsilon \in[0,2]$. Observe that every $p$-uniformly convex is uniformly convex. One should note that no Banach space is $p$-uniformly convex for $1<p<2$.

Remark 2.1 The basic properties of $E$, $J$, and $J^{-1}$ are as follows (see [24]). 
- If $E$ is an arbitrary Banach space, then $J$ is monotone and bounded;

- If $E$ is strictly convex, then $J$ is strictly monotone;

- If $E$ is smooth, then $J$ is single-valued and semi-continuous;

- If $E$ is uniformly smooth, then $J$ is uniformly norm-to-norm continuous on each bounded subset of $E$;

- If $E$ is reflexive smooth and strictly convex, then the normalized duality mapping $J$ is single-valued, one-to-one, and onto;

- If $E$ is a reflexive strictly convex and smooth Banach space and $J$ is the duality mapping from $E$ into $E^{*}$, then $J^{-1}$ is also single-valued, bijective and is also the duality mapping from $E^{*}$ into $E$, and thus $J J^{-1}=I_{E^{*}}$ and $J^{-1} J=I_{E}$;

- If $E$ is uniformly smooth, then $E$ is smooth and reflexive;

- If $E$ is a reflexive and strictly convex Banach space, then $J^{-1}$ is norm-weak"-continuous.

Remark 2.2 If $E$ is a reflexive strictly convex and smooth Banach space, then $\phi(x, y)=0$ if and only if $x=y$. It is sufficient to show that if $\phi(x, y)=0$, then $x=y$. From (1.5), we have $\|x\|=\|y\|$. This implies that $\langle x, J y\rangle=\|x\|^{2}=\|J y\|^{2}$. From the definition of $J$, one has $J x=J y$. Therefore, we have $x=y$ (see [24-26] for more details).

Recall that a Banach space $E$ has the Kadec-Klee property $[24,25,27]$ if for any sequence $\left\{x_{n}\right\} \subset E$ and $x \in E$ with $x_{n} \rightarrow x$ and $\left\|x_{n}\right\| \rightarrow\|x\|$, then $\left\|x_{n}-x\right\| \rightarrow 0$ as $n \rightarrow \infty$. It is well known that if $E$ is a uniformly convex Banach space, then $E$ has the Kadec-Klee property.

The generalized projection [15] from $E$ into $C$ is defined by $\Pi_{C}(x)=\operatorname{argmin}_{y \in C} \phi(y, x)$. The existence and uniqueness of the operator $\Pi_{C}$ follow from the properties of the functional $\phi(y, x)$ and the strict monotonicity of the mapping $J$ (see, for example, $[4,15,24,25$, 28]). If $E$ is a Hilbert space, then $\phi(x, y)=\|x-y\|^{2}$ and $\Pi_{C}$ becomes the metric projection $P_{C}: H \rightarrow C$. If $C$ is a nonempty closed and convex subset of a Hilbert space $H$, then $P_{C}$ is nonexpansive. This fact actually characterizes Hilbert spaces and consequently, it is not available in more general Banach spaces. We also need the following lemmas for the proof of our main results.

Lemma 2.3 (Alber [15]) Let $C$ be a nonempty closed convex subset of a smooth Banach space $E$ and let $x \in E$. Then $x_{0}=\Pi_{C} x$ if and only if

$$
\left\langle x_{0}-y, J x-J x_{0}\right\rangle \geq 0, \quad \forall y \in C .
$$

Lemma 2.4 (Alber [15]) Let E be a reflexive strictly convex and smooth Banach space, $C$ be a nonempty closed convex subset of $E$ and let $x \in E$. Then

$$
\phi\left(y, \Pi_{C} x\right)+\phi\left(\Pi_{C} x, x\right) \leq \phi(y, x), \quad \forall y \in C .
$$

Lemma 2.5 (Change et al. [22]) Let $C$ be a nonempty closed and convex subset of a uniformly smooth and strictly convex Banach space $E$ with the Kadec-Klee property. Let $S: C \rightarrow C$ be a closed and total quasi- $\phi$-asymptotically nonexpansive mapping with nonnegative real sequences $v_{n}$ and $\mu_{n}$ with $v_{n} \rightarrow 0, \mu_{n} \rightarrow 0$ as $n \rightarrow \infty$ and a strictly increasing continuous function $\zeta: \mathbb{R}^{+} \rightarrow \mathbb{R}^{+}$with $\zeta(0)=0$. If $\mu_{n}=0$, then the fixed point set $F(S)$ is a closed convex subset of $C$. 
For solving the equilibrium problem for a bifunction $f: C \times C \rightarrow \mathbb{R}$, let us assume that $f$ satisfies the following conditions:

(A1) $f(x, x)=0$ for all $x \in C$;

(A2) $f$ is monotone, i.e., $f(x, y)+f(y, x) \leq 0$ for all $x, y \in C$;

(A3) for each $x, y, z \in C$,

$$
\lim _{t \downarrow 0} f(t z+(1-t) x, y) \leq f(x, y)
$$

(A4) for each $x \in C, y \mapsto f(x, y)$ is convex and lower semi-continuous.

The following result is in Blum and Oettli [8].

Lemma 2.6 (Blum and Oettli [8]) Let $C$ be a closed convex subset of a smooth, strictly convex, and reflexive Banach space $E$, let $f$ be a bifunction from $C \times C$ to $\mathbb{R}$ satisfying (A1)-(A4), and let $r>0$ and $x \in E$. Then there exists $z \in C$ such that

$$
f(z, y)+\frac{1}{r}\langle y-z, J z-J x\rangle \geq 0, \quad \forall y \in C .
$$

Lemma 2.7 (Takahashi and Zembayashi [11]) Let $C$ be a closed convex subset of a uniformly smooth strictly convex and reflexive Banach space $E$ and let $f$ be a bifunction from $C \times C$ to $\mathbb{R}$ satisfying conditions (A1)-(A4). For all $r>0$ and $x \in E$, define a mapping $K_{r}: E \rightarrow C$ as follows:

$$
K_{r} x=\left\{z \in C: f(z, y)+\frac{1}{r}\langle y-z, J z-J x\rangle \geq 0, \forall y \in C\right\} .
$$

Then the following hold:

(1) $K_{r}$ is single-valued;

(2) $K_{r}$ is a firmly nonexpansive-type mapping [29], that is, for all $x, y \in E$,

$$
\left\langle K_{r} x-K_{r} y, J K_{r} x-J K_{r} y\right\rangle \leq\left\langle K_{r} x-K_{r} y, J x-J y\right\rangle ;
$$

(3) $F\left(K_{r}\right)=E P(f)$;

(4) $E P(f)$ is closed and convex.

Lemma 2.8 (Takahashi and Zembayashi [11]) Let C be a closed convex subset of a smooth strictly convex and reflexive Banach space $E$, let $f$ be a bifunction from $C \times C$ to $\mathbb{R}$ satisfying (A1)-(A4) and let $r>0$. Then, for $x \in E$ and $q \in F\left(K_{r}\right)$,

$$
\phi\left(q, K_{r} x\right)+\phi\left(K_{r} x, x\right) \leq \phi(q, x) .
$$

Lemma 2.9 [30] Let E be a uniformly convex Banach space and $B_{r}(0)=\{x \in E:\|x\| \leq r\}$ be a closed ball of $E$. Then there exists a continuous strictly increasing convex function $g:[0, \infty) \rightarrow[0, \infty)$ with $g(0)=0$ such that

$$
\|\lambda x+\mu y+\gamma z\|^{2} \leq\|\lambda x\|^{2}+\mid \mu y\left\|^{2}+\right\| \gamma z \|^{2}-\lambda \mu g(\|x-y\|)
$$

for all $x, y, z \in B_{r}(0)$ and $\lambda, \mu, \gamma \in[0,1]$ with $\lambda+\mu+\gamma=1$. 
Let $E$ be a smooth strictly convex and reflexive Banach space, $C$ be a nonempty closed convex subset of $E$ and $A \subset E \times E^{*}$ be a monotone operator satisfying $D(A) \subset C \subset$ $J^{-1}\left(\bigcap_{\lambda>0} R(J+\lambda A)\right)$. Then the resolvent $J_{\lambda}: C \rightarrow D(A)$ of $A$ is defined by

$$
J_{\lambda} x=\{z \in D(A): J x \in J z+\lambda A z, \forall x \in C\} .
$$

$J_{\lambda}$ is a single-valued mapping from $E$ to $D(A)$. For any $\lambda>0$, the Yosida approximation $A_{\lambda}: C \rightarrow E^{*}$ of $A$ is defined by $A_{\lambda} x=\frac{J x-J \lambda_{\lambda} x}{\lambda}$ for all $x \in C$. We know that $A_{\lambda} x \in A\left(J_{\lambda} x\right)$ for all $\lambda>0$ and $x \in E$.

Lemma 2.10 (Kohsaka and Takahashi [29]) Let E be a smooth strictly convex and reflexive Banach space, $C$ be a nonempty closed convex subset of $E$ and $A \subset E \times E^{*}$ be a monotone operator satisfying $D(A) \subset C \subset J^{-1}\left(\bigcap_{\lambda>0} R(J+\lambda A)\right)$. For any $\lambda>0$, let $J_{\lambda}$ and $A_{\lambda}$ be the resolvent and the Yosida approximation of $A$, respectively. Then the following hold:

(a) $\phi\left(p, J_{\lambda} x\right)+\phi\left(J_{\lambda} x, x\right) \leq \phi(p, x)$ for all $x \in C$ and $p \in A^{-1} 0$;

(b) $\left(J_{\lambda} x, A_{\lambda} x\right) \in A$ for all $x \in C$;

(c) $F\left(J_{\lambda}\right)=A^{-1} 0$.

Lemma 2.11 (Rockafellar [31]) Let E be a reflexive strictly convex and smooth Banach space. Then an operator $A \subset E \times E^{*}$ is maximal monotone if and only if $R(J+\lambda A)=E^{*}$ for all $\lambda>0$.

\section{Main result}

Theorem 3.1 Let $C$ be a nonempty closed and convex subset of a uniformly smooth and strictly uniformly convex Banach space $E$ with the Kadec-Klee property. Let $f$ be a bifunction from $C \times C$ to $\mathbb{R}$ satisfying the conditions (A1)-(A4) and let $A \subset E \times E^{*}$ be a maximal monotone operator satisfying $D(A) \subset C$ and $J_{r_{n}}=\left(J+r_{n} A\right)^{-1} J$ for all $r_{n}>0$. Let $S: C \rightarrow C$ be a closed and total quasi- $\phi$-asymptotically nonexpansive mapping with nonnegative real sequences $v_{n}^{S}, \mu_{n}^{S}$ with $v_{n}^{S} \rightarrow 0, \mu_{n}^{S} \rightarrow 0$ as $n \rightarrow \infty$ and a strictly increasing continuous function $\psi^{S}: \mathbb{R}^{+} \rightarrow \mathbb{R}^{+}$with $\psi^{S}(0)=0$. Let $T: C \rightarrow C$ be a closed and total quasi- $\phi$-asymptotically nonexpansive mapping with nonnegative real sequences $v_{n}^{T}, \mu_{n}^{T}$ with $v_{n}^{T} \rightarrow 0, \mu_{n}^{T} \rightarrow 0$ as $n \rightarrow \infty$ and a strictly increasing continuous function $\psi^{T}: \mathbb{R}^{+} \rightarrow \mathbb{R}^{+}$with $\psi^{T}(0)=0$. Assume that $S$ and $T$ are uniformly L-Lipschitz continuous and $F=F(S) \cap F(T) \cap E P(f) \cap A^{-1} 0 \neq \emptyset$. For an initial point $x_{1} \in E, C_{1}=C$, define the sequence $\left\{x_{n}\right\}$ by

$$
\left\{\begin{array}{l}
z_{n}=J_{r_{n}} x_{n}, \\
u_{n}=K_{r_{n}} x_{n}, \\
y_{n}=J^{-1}\left(\alpha_{n} J x_{n}+\beta_{n} J S^{n} z_{n}+\gamma_{n} J T^{n} u_{n}\right), \\
C_{n+1}=\left\{v \in C_{n}: \phi\left(v, y_{n}\right) \leq \phi\left(v, x_{n}\right)+\zeta_{n}\right\}, \\
x_{n+1}=\Pi_{C_{n+1}} x_{1}, \quad n \in \mathbb{N},
\end{array}\right.
$$

where $\left\{\alpha_{n}\right\},\left\{\beta_{n}\right\}$, and $\left\{\gamma_{n}\right\}$ are sequences in $(0,1)$ such that $\alpha_{n}+\beta_{n}+\gamma_{n}=1,\left\{r_{n}\right\} \subset[d, \infty)$ for some $d>0 \mu_{n}=\sup \left\{\mu_{n}^{S}, \mu_{n}^{T}\right\}, v_{n}=\sup \left\{v_{n}^{S}, v_{n}^{T}\right\}, \psi=\sup \left\{\psi^{S}, \psi^{T}\right\}$ for all $n \geq 1, \zeta=$ $v_{n} \sup _{q \in \mathcal{F}} \psi\left(\phi\left(q, x_{n}\right)\right)+\mu_{n}$. If $\lim _{n \rightarrow \infty} \alpha_{n} \beta_{n}=0$ and $\liminf _{n \rightarrow \infty} \alpha_{n} \gamma_{n}<1$, then $\left\{x_{n}\right\}$ converges strongly to $\Pi_{F} x_{1}$. 
Proof First, we show that $C_{n}$ is closed and convex for all $n \in \mathbb{N}$ since $C_{1}=C$ is convex. Suppose that $C_{n}$ is convex for all $n \in \mathbb{N}$. For any $v \in C_{n}$, we know that $\phi\left(v, y_{n}\right) \leq \phi\left(v, x_{n}\right)+\zeta_{n}$ is equivalent to

$$
2\left\langle v, J x_{n}-J y_{n}\right\rangle \leq\left\|x_{n}\right\|^{2}-\left\|y_{n}\right\|^{2}+\zeta_{n} .
$$

That is, $C_{n+1}$ is convex for all $n \in \mathbb{N}$. By the definition of $C_{n}$, it is obvious that $C_{n}$ is closed for all $n \in \mathbb{N}$.

We show that $\left\{x_{n}\right\}$ is well defined. It is obvious that $F \subset C_{1}=C$. Suppose $F \subset C_{n}$ for $n \in \mathbb{N}$, from Lemma 2.8 and Lemma $2.10, S, T$ are total quasi- $\phi$-asymptotically nonexpansive mappings. For each $q \in F \subset C_{n}$, it follows that

$$
\begin{aligned}
\phi\left(q, y_{n}\right)= & \phi\left(q, J^{-1}\left(\alpha_{n} J x_{n}+\beta_{n} J S^{n} z_{n}+\gamma_{n} J T^{n} u_{n}\right)\right) \\
= & \|q\|^{2}-2\left|q, \alpha_{n} J x_{n}+\beta_{n} J S^{n} z_{n}+\gamma_{n} J T^{n} u_{n}\right\rangle+\left\|\alpha_{n} J x_{n}+\beta_{n} J S^{n} z_{n}+\gamma_{n} J T^{n} u_{n}\right\|^{2} \\
\leq & \alpha_{n} \phi\left(q, x_{n}\right)+\beta_{n} \phi\left(q, S^{n} z_{n}\right)+\gamma_{n} \phi\left(q, T^{n} u_{n}\right) \\
\leq & \alpha_{n} \phi\left(q, x_{n}\right)+\beta_{n}\left(\phi\left(q, z_{n}\right)+v_{n}^{S} \psi^{S}\left(\phi\left(q, z_{n}\right)\right)+\mu_{n}^{S}\right) \\
& +\gamma_{n}\left(\phi\left(q, u_{n}\right)+v_{n}^{T} \psi^{T}\left(\phi\left(q, u_{n}\right)\right)+\mu_{n}^{T}\right) \\
= & \alpha_{n} \phi\left(q, x_{n}\right)+\beta_{n} \phi\left(q, z_{n}\right)+\beta_{n} v_{n}^{S} \psi^{S}\left(\phi\left(q, z_{n}\right)\right)+\beta_{n} \mu_{n}^{S} \\
& +\gamma_{n} \phi\left(q, u_{n}\right)+\gamma_{n} v_{n}^{T} \psi^{T}\left(\phi\left(q, u_{n}\right)\right)+\gamma_{n} \mu_{n}^{T} \\
\leq & \alpha_{n} \phi\left(q, x_{n}\right)+\beta_{n} \phi\left(q, z_{n}\right)+\beta_{n} v_{n}^{S} \psi^{S}\left(\phi\left(q, x_{n}\right)\right)+\beta_{n} \mu_{n}^{S} \\
& +\gamma_{n} \phi\left(q, u_{n}\right)+\gamma_{n} v_{n}^{T} \psi^{T}\left(\phi\left(q, x_{n}\right)\right)+\gamma_{n} \mu_{n}^{T} \\
\leq & \alpha_{n} \phi\left(q, x_{n}\right)+\beta_{n} \phi\left(q, z_{n}\right)+\beta_{n} v_{n} \psi\left(\phi\left(q, x_{n}\right)\right)+\beta_{n} \mu_{n} \\
& +\gamma_{n} \phi\left(q, u_{n}\right)+\gamma_{n} v_{n} \psi\left(\phi\left(q, x_{n}\right)\right)+\gamma_{n} \mu_{n} \\
\leq & \alpha_{n} \phi\left(q, x_{n}\right)+\beta_{n} \phi\left(q, z_{n}\right)+\gamma_{n} \phi\left(q, u_{n}\right)+\left(1-\alpha_{n}\right) v_{n} \psi\left(\phi\left(q, x_{n}\right)\right)+\left(1-\alpha_{n}\right) \mu_{n} \\
\leq & \alpha_{n} \phi\left(q, x_{n}\right)+\beta_{n} \phi\left(q, z_{n}\right)+\gamma_{n} \phi\left(q, u_{n}\right)+v_{n} \sup _{q \in F} \psi\left(\phi\left(q, x_{n}\right)\right)+\mu_{n} \\
\leq & \alpha_{n} \phi\left(q, x_{n}\right)+\beta_{n} \phi\left(q, z_{n}\right)+\gamma_{n} \phi\left(q, u_{n}\right)+\zeta_{n} \\
\leq & \alpha_{n} \phi\left(q, x_{n}\right)+\beta_{n} \phi\left(q, x_{n}\right)+\gamma_{n} \phi\left(q, u_{n}\right)+\zeta_{n} \\
\leq & \alpha_{n} \phi\left(q, x_{n}\right)+\beta_{n} \phi\left(q, x_{n}\right)+\gamma_{n} \phi\left(q, K_{r_{n}} x_{n}\right)+\zeta_{n} \\
\leq & \alpha_{n} \phi\left(q, x_{n}\right)+\beta_{n} \phi\left(q, x_{n}\right)+\gamma_{n} \phi\left(q, x_{n}\right)+\zeta_{n} \\
\leq & \phi\left(q, x_{n}\right)+\zeta_{n}, \\
&
\end{aligned}
$$

where $\zeta_{n}=v_{n} \sup _{q \in F} \psi\left(\phi\left(q, x_{n}\right)\right)+\mu_{n}$. This shows that $q \in C_{n+1}$, thus $F \subset C_{n+1}$. Hence, $F \subset$ $C_{n}$ for all $n \geq 1$. This implies that the sequence $\left\{x_{n}\right\}$ is well defined.

We show that $\lim _{n \rightarrow \infty} x_{n}=p$. From the definition of $C_{n+1}$ with $x_{n}=\Pi_{C_{n}} x_{1}$ and $x_{n+1}=$ $\Pi_{C_{n+1}} x_{1} \in C_{n+1} \subset C_{n}$, it follows that

$$
\phi\left(x_{n}, x_{1}\right) \leq \phi\left(x_{n+1}, x_{1}\right), \quad \forall n \geq 1
$$


By Lemma 2.4, we get

$$
\begin{aligned}
\phi\left(x_{n}, x_{1}\right) & =\phi\left(\Pi_{C_{n}} x_{1}, x_{1}\right) \\
& \leq \phi\left(q, x_{1}\right)-\phi\left(q, x_{n}\right) \\
& \leq \phi\left(q, x_{1}\right), \quad \forall q \in F .
\end{aligned}
$$

From (3.3) and (3.4), we have that $\lim _{n \rightarrow \infty} \phi\left(x_{n}, x_{1}\right)$ exists. In particular, it follows from (1.6) that the sequence $\left\{x_{n}\right\}$ is bounded and so are $\left\{z_{n}\right\},\left\{u_{n}\right\}$, and $\left\{y_{n}\right\}$. Since $x_{n} \in C_{n} \subset E$ and $E$ is reflexive, the sequence $\left\{x_{n}\right\}$ converges weakly to an element of $E$, we assume that $x_{n} \rightarrow p$. Note that $C_{n}$ is closed and convex and $x_{n} \in C_{n}$. We have that $p \in C_{n}$, that is,

$$
x_{n} \rightarrow p \in C_{n} \quad \text { as } n \rightarrow \infty \text {. }
$$

For $p \in C_{n}$, we have

$$
\begin{aligned}
\liminf _{n \rightarrow \infty} \phi\left(x_{n}, x_{1}\right) & =\liminf _{n \rightarrow \infty}\left\{\left\|x_{n}\right\|^{2}-2\left\langle x_{n}, J x_{1}\right\rangle+\left\|x_{1}\right\|^{2}\right\} \\
& \geq\|p\|^{2}-2\left\langle p, J x_{1}\right\rangle+\left\|x_{1}\right\|^{2} \\
& =\phi\left(p, x_{1}\right) .
\end{aligned}
$$

On the other hand, $x_{n}=\Pi_{C_{n}} x_{1}$, we have

$$
\phi\left(x_{n}, x_{1}\right) \leq \phi\left(p, x_{1}\right), \quad \forall p \in C_{n} .
$$

It follows that

$$
\phi\left(p, x_{1}\right) \leq \liminf _{n \rightarrow \infty} \phi\left(x_{n}, x_{1}\right) \leq \limsup _{n \rightarrow \infty} \phi\left(x_{n}, x_{1}\right) \leq \phi\left(p, x_{1}\right)
$$

This implies that $\lim _{n \rightarrow \infty} \phi\left(x_{n}, x_{1}\right)=\phi\left(p, x_{1}\right)$. Hence, we get

$$
\left\|x_{n}\right\| \rightarrow\|p\| \quad \text { as } n \rightarrow \infty
$$

From (3.5), (3.6), and the Kadec-Klee property of $E$, we have

$$
\lim _{n \rightarrow \infty} x_{n}=p
$$

Therefore,

$$
\lim _{n \rightarrow \infty} \zeta_{n}=\lim _{n \rightarrow \infty} v_{n} \sup _{q \in \mathcal{F}} \psi\left(\phi\left(q, x_{n}\right)\right)+\mu_{n}=0 .
$$

From (3.7), it follows that

$$
\lim _{n \rightarrow \infty}\left\|x_{n}-x_{n+1}\right\|=0
$$


and hence

$$
\lim _{n \rightarrow \infty}\left\|J x_{n}-J x_{n+1}\right\|=0 .
$$

We show that $p \in F(S) \cap F(T) \cap A^{-1} 0 \cap E P(f)$.

Now, we show that $p \in E P(f)$. For $x_{n+1} \in C_{n+1} \subset C_{n}$ and $x_{n}=\Pi_{C_{n}} x_{1}$, it follows that

$$
\begin{aligned}
\phi\left(x_{n+1}, x_{n}\right) & =\phi\left(x_{n+1}, \Pi_{C_{n}} x_{1}\right) \\
& \leq \phi\left(x_{n+1}, x_{1}\right)-\phi\left(\Pi_{C_{n}} x_{1}, x_{1}\right) \\
& =\phi\left(x_{n+1}, x_{1}\right)-\phi\left(x_{n}, x_{1}\right) .
\end{aligned}
$$

Since $\lim _{n \rightarrow \infty} \phi\left(x_{n}, x_{1}\right)$ exists, we have

$$
\lim _{n \rightarrow \infty} \phi\left(x_{n+1}, x_{n}\right)=0
$$

Since $x_{n+1} \subset C_{n}$ and the definition of $C_{n+1}$, we have $\phi\left(x_{n+1}, y_{n}\right) \leq \phi\left(x_{n+1}, x_{n}\right)+\zeta_{n}$. From (3.11), we also have

$$
\lim _{n \rightarrow \infty} \phi\left(x_{n+1}, y_{n}\right)=0
$$

From (1.6) and (3.7), it follows that

$$
\left\|y_{n}\right\| \rightarrow\|p\| \quad \text { as } n \rightarrow \infty,
$$

and hence

$$
\left\|y_{n}\right\| \rightarrow\|J p\| \quad \text { as } n \rightarrow \infty .
$$

This implies that $\left\{\left\|J y_{n}\right\|\right\}$ is bounded. Note that $E$ is reflexive and $E^{*}$ is also reflexive, we can assume that $J y_{n} \rightarrow x^{*} \in E^{*}$. Since $E$ is reflexive, we see that $J(E)=E^{*}$. Hence, there exists $x \in E$ such that $J x=x^{*}$ and we have

$$
\begin{aligned}
\phi\left(x_{n+1}, y_{n}\right) & =\left\|x_{n+1}\right\|^{2}-2\left\langle x_{n+1}, J y_{n}\right\rangle+\left\|y_{n}\right\|^{2} \\
& =\left\|x_{n+1}\right\|^{2}-2\left\langle x_{n+1}, J y_{n}\right\rangle+\left\|y_{n}\right\|^{2} .
\end{aligned}
$$

Taking $\liminf _{n \rightarrow \infty}$ on the both sides of the equality above, in view of the weak lower semicontinuity of the norm $\|\cdot\|$, it follows that

$$
\begin{aligned}
0 & \geq\|p\|^{2}-2\left\langle p, x^{*}\right\rangle+\left\|x^{*}\right\|^{2} \\
& =\|p\|^{2}-2\langle p, J x\rangle+\|J\|^{2} \\
& =\|p\|^{2}-2\langle p, J x\rangle+\|x\|^{2} \\
& =\phi(p, x) .
\end{aligned}
$$


From Remark 2.2, we have $p=x$, which implies that $J y_{n} \rightarrow J p$ as $n \rightarrow \infty$. From the KadecKlee property of $E^{*}$, we obtain that

$$
J y_{n} \rightarrow J p \quad \text { as } n \rightarrow \infty .
$$

Note that $J^{-1}: E^{*} \rightarrow E$ is demicontinuous, that is, $y_{n} \rightarrow p$ as $n \rightarrow \infty$. From the Kadec-Klee property of $E$, it follows that

$$
\lim _{n \rightarrow \infty} y_{n}=p
$$

From (3.2), (3.7), and (3.16), it follows that $\lim _{n \rightarrow \infty} \phi\left(q, u_{n}\right)=\phi(q, p)$. Since $u_{n}=K_{r_{n}} x_{n}$ and from Lemma 2.8 , we have

$$
\phi\left(u_{n}, x_{n}\right)=\phi\left(K_{r_{n}} x_{n}, x_{n}\right) \leq \phi\left(q, x_{n}\right)-\phi\left(q, K_{r_{n}} x_{n}\right)=\phi\left(q, x_{n}\right)-\phi\left(q, z_{n}\right) \rightarrow 0 \quad \text { as } n \rightarrow \infty .
$$

From (1.6), it follows that

$$
\left\|u_{n}\right\| \rightarrow\|p\| \quad \text { as } n \rightarrow \infty
$$

Since $\left\{u_{n}\right\}$ is bounded and $E$ is also reflexive, we can assume that $u_{n} \rightarrow u \in E$ and we have

$$
\phi\left(u_{n}, x_{n}\right)=\left\|u_{n}\right\|^{2}-2\left\langle u_{n}, J x_{n}\right\rangle+\left\|x_{n}\right\|^{2} .
$$

Taking $\liminf \operatorname{in}_{n \rightarrow \infty}$ on the both sides of the equality above, in view of the weak lower semicontinuity of the norm $\|\cdot\|$, it follows that

$$
\begin{aligned}
0 & \geq\|u\|^{2}-2\langle u, J p\rangle+\|p\|^{2} \\
& =\phi(u, p) .
\end{aligned}
$$

From Remark 2.2, we have $u=p$, that is, $u_{n} \rightarrow p$ as $n \rightarrow \infty$. From the Kadec-Klee property of $E$, we obtain that

$$
\lim _{n \rightarrow \infty} u_{n}=p
$$

Since $\lim _{n \rightarrow \infty} u_{n}=p$ and $\lim _{n \rightarrow \infty} x_{n}=p$, we have that

$$
\lim _{n \rightarrow \infty}\left\|u_{n}-x_{n}\right\|=0
$$

Since $J$ is uniformly norm-to-norm continuous, we obtain

$$
\lim _{n \rightarrow \infty}\left\|J u_{n}-J x_{n}\right\|=0 .
$$

From $r_{n}>0$, we have $\frac{\left\|u_{n}-J x_{n}\right\|}{r_{n}} \rightarrow 0$ as $n \rightarrow \infty$ and

$$
f\left(u_{n}, y\right)+\frac{1}{r_{n}}\left\langle y-u_{n}, J u_{n}-J x_{n}\right\rangle \geq 0, \quad \forall y \in C .
$$


By (A2),

$$
\begin{aligned}
\left\|y-u_{n}\right\| \frac{\left\|J u_{n}-J x_{n}\right\|}{r_{n}} & \geq \frac{1}{r_{n}}\left\langle y-u_{n}, J u_{n}-J x_{n}\right\rangle \\
& \geq-f\left(u_{n}, y\right) \\
& \geq f\left(y, u_{n}\right), \quad \forall y \in C,
\end{aligned}
$$

and $u_{n} \rightarrow p$, we get $f(y, p) \leq 0$ for all $y \in C$. For $0<t<1$, define $y_{t}=t y+(1-t) p$. Then $y_{t} \in C$, which implies that $f\left(y_{t}, p\right) \leq 0$. From (A1), we obtain that

$$
0=f\left(y_{t}, y_{t}\right) \leq t f\left(y_{t}, y\right)+(1-t) f\left(y_{t}, p\right) \leq t f\left(y_{t}, y\right) .
$$

Thus $f\left(y_{t}, y\right) \geq 0$. From (A3), we have $f(p, y) \geq 0$ for all $y \in C$. Hence, $p \in E P(f)$.

Next, we show that $p \in A^{-1} 0$. From (3.2), (3.7), (3.16), and (3.18), it follows that $\lim _{n \rightarrow \infty} \phi\left(q, z_{n}\right)=\phi(q, p)$. Since $z_{n}=J_{r_{n}} x_{n}$ and from Lemma 2.10, we have

$$
\phi\left(z_{n}, x_{n}\right)=\phi\left(J_{r_{n}} x_{n}, x_{n}\right) \leq \phi\left(q, x_{n}\right)-\phi\left(q, J_{r_{n}} x_{n}\right)=\phi\left(q, x_{n}\right)-\phi\left(q, z_{n}\right) \rightarrow 0 \quad \text { as } n \rightarrow \infty .
$$

From (1.6), it follows that

$$
\left\|z_{n}\right\| \rightarrow\|p\| \quad \text { as } n \rightarrow \infty
$$

Since $\left\{u_{n}\right\}$ is bounded and $E$ is also reflexive, we can assume that $z_{n} \rightarrow z \in E$ and we have

$$
\phi\left(z_{n}, x_{n}\right)=\left\|z_{n}\right\|^{2}-2\left\langle z_{n}, J x_{n}\right\rangle+\left\|x_{n}\right\|^{2} .
$$

Taking $\liminf _{n \rightarrow \infty}$ on the both sides of the equality above, in view of the weak lower semicontinuity of the norm $\|\cdot\|$, it follows that

$$
\begin{aligned}
0 & \geq\|z\|^{2}-2\langle z, J p\rangle+\|p\|^{2} \\
& =\phi(z, p) .
\end{aligned}
$$

From Remark 2.2, we have $z=p$, that is, $u_{n} \rightarrow p$ as $n \rightarrow \infty$. From the Kadec-Klee property of $E$, we obtain that

$$
\lim _{n \rightarrow \infty} z_{n}=p
$$

Since $\lim _{n \rightarrow \infty} z_{n}=p$ and $\lim _{n \rightarrow \infty} x_{n}=p$, we have that

$$
\lim _{n \rightarrow \infty}\left\|z_{n}-x_{n}\right\|=0
$$

and hence

$$
\lim _{n \rightarrow \infty}\left\|J x_{n}-J z_{n}\right\|=0
$$


From the condition $\left\{r_{n}\right\} \subset[d, \infty)$ for some $d>0$, we have

$$
\lim _{n \rightarrow \infty} \frac{1}{r_{n}}\left\|J x_{n}-J z_{n}\right\|=0
$$

Thus, since $z_{n}=J_{r_{n}} x_{n}$, we have

$$
\lim _{n \rightarrow \infty}\left\|A_{r_{n}} x_{n}\right\|=\lim _{n \rightarrow \infty} \frac{1}{r_{n}}\left\|J x_{n}-J z_{n}\right\|=0
$$

For any $\left(w, w^{*}\right) \in G(A)$, it follows from the monotonicity of $A$ that $\left\langle w-z_{n}, w^{*}-A_{r_{n}} x_{n}\right\rangle \geq$ 0 for all $n \geq 0$. Letting $n \rightarrow \infty$, we get $\left\langle w-p, w^{*}\right\rangle \geq 0$. Therefore, since $A$ is maximal monotone, we obtain $p \in A^{-1} 0$.

On the other hand, we have

$$
\begin{aligned}
\phi\left(q, x_{n}\right)-\phi\left(q, y_{n}\right) & =\left\|x_{n}\right\|^{2}-\left\|y_{n}\right\|^{2}-2\left\langle q, J x_{n}-J u_{n}\right\rangle \\
& \leq\left\|x_{n}-y_{n}\right\|\left(\left\|x_{n}+y_{n}\right\|\right)+2\|q\|\left\|J x_{n}-J y_{n}\right\| .
\end{aligned}
$$

In view of $\left\|x_{n}-y_{n}\right\| \rightarrow 0$ and $\left\|J x_{n}-J y_{n}\right\| \rightarrow 0$ as $n \rightarrow \infty$, we obtain that

$$
\phi\left(q, x_{n}\right)-\phi\left(q, y_{n}\right) \rightarrow 0 \quad \text { as } n \rightarrow \infty .
$$

From Lemma 2.9, we have

$$
\begin{aligned}
\phi\left(q, y_{n}\right)= & \phi\left(q, J^{-1} \alpha_{n} J x_{n}+\beta_{n} J S^{n} z_{n}+\gamma_{n} J T^{n} u_{n}\right) \\
\leq & \left.\|q\|^{2}-2\left\langle q, \alpha_{n} J x_{n}+\beta_{n} J S^{n} z_{n}+\gamma_{n} J T^{n} u_{n}\right\rangle+\| \alpha_{n} J x_{n}+\beta_{n} J S^{n} z_{n}+\gamma_{n} J T^{n} u_{n}\right\rangle \|^{2} \\
& -\alpha_{n} \beta_{n} g\left(\left\|J x_{n}-J S^{n} z_{n}\right\|\right) \\
= & \alpha_{n} \phi\left(q, x_{n}\right)+\beta_{n} \phi\left(q, S^{n} z_{n}\right)+\gamma_{n} \phi\left(q, T^{n} u_{n}\right)-\alpha_{n} \beta_{n} g\left(\left\|J x_{n}-J S^{n} z_{n}\right\|\right) \\
\leq & \phi\left(q, x_{n}\right)+\zeta_{n}-\alpha_{n} \beta_{n} g\left(\left\|J x_{n}-J S^{n} z_{n}\right\|\right) .
\end{aligned}
$$

It follows from $\liminf _{n \rightarrow \infty} \alpha_{n} \beta_{n}>0$, (3.23), (3.8), and the property of $g$ that

$$
\lim _{n \rightarrow \infty}\left\|J x_{n}-J S^{n} z_{n}\right\|=0
$$

Since $x_{n} \rightarrow p$ as $n \rightarrow \infty$ and $J$ is uniformly continuous, it yields that $J x_{n} \rightarrow J p$, we have

$$
J S^{n} z_{n} \rightarrow J p
$$

Since $J^{-1}$ is demicontinuous, we also have

$$
S^{n} z_{n} \rightarrow p
$$

On the other hand, we observe that

$$
\left|\left\|S^{n} z_{n}\right\|-\|p\|\right|=\left|\left\|J\left(S^{n} z_{n}\right)\right\|-\|J p\|\right| \leq\left\|J\left(S^{n} z_{n}\right)-J p\right\|,
$$


we obtain that $\left\|S^{n} z_{n}\right\| \rightarrow\|p\|$. Since $E$ has the Kadee-Klee property, we get

$$
\lim _{n \rightarrow \infty} S^{n} z_{n}=p
$$

By the assumption that $S$ is uniformly $L$-Lipschitz continuous, we have

$$
\begin{aligned}
\left\|S^{n+1} z_{n}-S^{n} z_{n}\right\| & \leq\left\|S^{n+1} z_{n}-S^{n+1} z_{n+1}\right\|+\left\|S^{n+1} z_{n+1}-z_{n+1}\right\|+\left\|z_{n+1}-z_{n}\right\|+\left\|z_{n}-S^{n} z_{n}\right\| \\
& \leq(L+1)\left\|z_{n+1}-z_{n}\right\|+\left\|S^{n+1} z_{n+1}-z_{n+1}\right\|+\left\|z_{n}-S^{n} z_{n}\right\| .
\end{aligned}
$$

Since $\lim _{n \rightarrow \infty} z_{n}=p$ and $\lim _{n \rightarrow \infty} S^{n} z_{n}=p$, it yields that $\left\|S^{n+1} z_{n}-S^{n} z_{n}\right\| \rightarrow 0, n \rightarrow \infty$. From $S^{n} z_{n} \rightarrow p$, we get $S^{n+1} z_{n} \rightarrow p$, that is, $S S^{n} z_{n} \rightarrow p$. In view of the closeness of $S$, we have $S p=p$. This implies that $p \in F(S)$. By the same way, we have that $p \in F(T)$.

We show that $p=\Pi_{F} x_{1}$. From $x_{n}=\Pi_{C_{n}} x_{1}$, we have $\left\langle J x_{1}-J x_{n}, x_{n}-v\right\rangle \geq 0, \forall v \in C_{n}$. Since $F \subset C_{n}$, we also have

$$
\left\langle J x_{1}-J x_{n}, x_{n}-y\right\rangle \geq 0, \quad \forall y \in F .
$$

By taking limit $n \rightarrow \infty$, we obtain that

$$
\left\langle x_{1}-J p, p-y\right\rangle \geq 0, \quad \forall y \in F .
$$

By Lemma 2.3, we can conclude that $p=\Pi_{F} x_{1}$ and $x_{n} \rightarrow p$ as $n \rightarrow \infty$. The proof is completed.

Let $A$ be a continuous and monotone operator of $C$ into $E^{*}$. Then we can find a solution of $V I(A, C)$ in a uniformly smooth and strictly convex Banach space $E$ with the Kadec-Klee property by using the following lemma.

Lemma 3.2 (Zegeye and Shahzad [32]) Let $C$ be a nonempty closed convex subset of a uniformly smooth strictly convex real Banach space E. Let $A: C \rightarrow E^{*}$ be a continuous monotone mapping. For any $r>0$, define a mapping $W_{r}: E \rightarrow C$ as follows:

$$
W_{r} x=\left\{z \in C:\langle y-z, A z\rangle+\frac{1}{r}\langle y-z, J z-J x\rangle \geq 0, \forall y \in C\right\}
$$

for all $x \in C$. Then the following hold:

(1) $W_{r}$ is single-valued;

(2) $F\left(W_{r}\right)=V I(A, C)$;

(3) $\operatorname{VI}(A, C)$ is a closed and convex subset of $C$;

(4) $\phi\left(q, W_{r} x\right)+\phi\left(W_{r} x, x\right) \leq \phi(q, x)$ for all $q \in F\left(W_{r}\right)$.

Corrollary 3.3 Let C be a nonempty closed and convex subset of a uniformly smooth and strictly uniformly convex Banach space $E$ with the Kadec-Klee property. Let $f$ be a bifunction from $C \times C$ to $\mathbb{R}$ satisfying the conditions (A1)-(A4) and let $A$ be a continuous and monotone operator of $C$ into $E^{*}$. Let $S: C \rightarrow C$ be a closed and total quasi- $\phi$-asymptotically nonexpansive mapping with nonnegative real sequences $v_{n}^{S}, \mu_{n}^{S}$ with $v_{n}^{S} \rightarrow 0, \mu_{n}^{S} \rightarrow 0$ as $n \rightarrow \infty$ and a strictly increasing continuous function $\psi^{S}: \mathbb{R}^{+} \rightarrow \mathbb{R}^{+}$with $\psi^{S}(0)=0$. Let 
$T: C \rightarrow C$ be a closed and total quasi- $\phi$-asymptotically nonexpansive mapping with nonnegative real sequences $v_{n}^{T}, \mu_{n}^{T}$ with $v_{n}^{T} \rightarrow 0, \mu_{n}^{T} \rightarrow 0$ as $n \rightarrow \infty$ and a strictly increasing continuous function $\psi^{T}: \mathbb{R}^{+} \rightarrow \mathbb{R}^{+}$with $\psi^{T}(0)=0$. Assume that $S$ and $T$ are uniformly L-Lipschitz continuous and $F=F(S) \cap F(T) \cap E P(f) \cap V I(A, C) \neq \emptyset$. For an initial point $x_{1} \in E, C_{1}=C$, define the sequence $\left\{x_{n}\right\}$ by

$$
\left\{\begin{array}{l}
z_{n}=W_{r_{n}} x_{n}, \\
u_{n}=K_{r_{n}} x_{n}, \\
y_{n}=J^{-1}\left(\alpha_{n} J x_{n}+\beta_{n} J S^{n} z_{n}+\gamma_{n} J T^{n} u_{n}\right), \\
C_{n+1}=\left\{v \in C_{n}: \phi\left(v, y_{n}\right) \leq \phi\left(v, x_{n}\right)+\zeta_{n}\right\}, \\
x_{n+1}=\Pi_{C_{n+1}} x_{1}, \quad n \in \mathbb{N},
\end{array}\right.
$$

where $\left\{\alpha_{n}\right\},\left\{\beta_{n}\right\}$ and $\left\{\gamma_{n}\right\}$ are sequences in $(0,1)$ such that $\alpha_{n}+\beta_{n}+\gamma_{n}=1,\left\{r_{n}\right\} \subset[d, \infty)$ for some $d>0 \mu_{n}=\sup \left\{\mu_{n}^{S}, \mu_{n}^{T}\right\}, v_{n}=\sup \left\{v_{n}^{S}, v_{n}^{T}\right\}, \psi=\sup \left\{\psi^{S}, \psi^{T}\right\}$ for all $n \geq 1, \zeta=$ $v_{n} \sup _{q \in \mathcal{F}} \psi\left(\phi\left(q, x_{n}\right)\right)+\mu_{n}$. If $\lim _{n \rightarrow \infty} \alpha_{n} \beta_{n}=0$ and $\liminf _{n \rightarrow \infty} \alpha_{n} \gamma_{n}<1$, then $\left\{x_{n}\right\}$ converges strongly to $\Pi_{F} x_{1}$.

Proof From the proof of Theorem 3.1, we known that $\lim _{n \rightarrow \infty} z_{n}=p$ and $\lim _{n \rightarrow \infty} x_{n}=p$. We obtain that

$$
\lim _{n \rightarrow \infty}\left\|z_{n}-x_{n}\right\|=0
$$

and hence

$$
\lim _{n \rightarrow \infty}\left\|J x_{n}-J z_{n}\right\|=0
$$

Since $\left\{r_{n}\right\} \subset[d, \infty)$ for some $d>0$, we have

$$
\lim _{n \rightarrow \infty} \frac{1}{r_{n}}\left\|J x_{n}-J z_{n}\right\|=0 .
$$

From the definition of $W$, it follows that

$$
\left\langle y-z_{n}, A_{n} z_{n}\right\rangle+\frac{1}{r_{n}}\left\langle y-z_{n}, J z_{n}-J x_{n}\right\rangle \geq 0, \quad \forall y \in C .
$$

For $0<t<1$, define $y_{t}=t y+(1-t) p$, then $y_{t} \in C$. We have

$$
\left\langle y_{t}-z_{n}, A_{n} z_{n}\right\rangle+\frac{1}{r_{n}}\left\langle y_{t}-z_{n}, J z_{n}-J x_{n}\right\rangle \geq 0, \quad \forall y_{t} \in C .
$$

It follows that

$$
\begin{aligned}
\left\langle y_{t}-z_{n}, A_{n} y_{t}\right\rangle & \geq\left\langle y_{t}-z_{n}, A_{n} y_{t}\right\rangle-\left\langle y_{t}-z_{n}, A_{n} z_{n}\right\rangle+\frac{1}{r_{n}}\left\langle y_{t}-z_{n}, J z_{n}-J x_{n}\right\rangle \\
& =\left\langle y_{t}-z_{n}, A_{n} y_{t}-A_{n} z_{n}\right\rangle+\left\langle y_{t}-z_{n}, \frac{J z_{n}-J x_{n}}{r_{n}}\right\rangle .
\end{aligned}
$$


Since $A_{n}$ are continuous and monotone mappings, we have $\left\langle y_{t}-z_{n}, A_{n} y_{t}-A_{n} z_{n}\right\rangle \geq 0$. From (3.31) and (3.32), it follows that $\left\langle y_{t}-z_{n}, A_{n} y_{t}\right\rangle \geq 0$. Take the limit as $n \rightarrow \infty$ and $z_{n} \rightarrow p$. We get $\left\langle y_{t}-p, A_{n} y_{t}\right\rangle \geq 0$ for all $y_{t} \in C$. Therefore, $\left\langle y-p, A_{n} y_{t}\right\rangle \geq 0$ for all $y \in C$. If $t \rightarrow 0$, we have that $\left\langle y-p, A_{n} p\right\rangle \geq 0$ for all $y \in C$. Hence, $p \in V I(A, C)$. The proof is completed.

Corrollary 3.4 Let C be a nonempty closed and convex subset of a uniformly smooth and strictly uniformly convex Banach space $E$ with the Kadec-Klee property. Let $A$ be a continuous and monotone operator of $C$ into $E^{*}$ and let $B \subset E \times E^{*}$ be a maximal monotone operator satisfying $D(B) \subset C$ and $J_{r_{n}}=\left(J+r_{n} B\right)^{-1} J$ for all $r_{n}>0$. Let $S: C \rightarrow C$ be a closed and total quasi- $\phi$-asymptotically nonexpansive mapping with nonnegative real sequences $v_{n}^{S}, \mu_{n}^{S}$ with $v_{n}^{S} \rightarrow 0, \mu_{n}^{S} \rightarrow 0$ as $n \rightarrow \infty$ and a strictly increasing continuous function $\psi^{S}: \mathbb{R}^{+} \rightarrow$ $\mathbb{R}^{+}$with $\psi^{S}(0)=0$. Let $T: C \rightarrow C$ be a closed and total quasi- $\phi$-asymptotically nonexpansive mapping with nonnegative real sequences $v_{n}^{T}, \mu_{n}^{T}$ with $v_{n}^{T} \rightarrow 0, \mu_{n}^{T} \rightarrow 0$ as $n \rightarrow \infty$ and a strictly increasing continuous function $\psi^{T}: \mathbb{R}^{+} \rightarrow \mathbb{R}^{+}$with $\psi^{T}(0)=0$. Assume that $S$ and $T$ are uniformly L-Lipschitz continuous and $F=F(S) \cap F(T) \cap V I(A, C) \cap B^{-1} 0 \neq \emptyset$. For an initial point $x_{1} \in E, C_{1}=C$, define the sequence $\left\{x_{n}\right\}$ by

$$
\left\{\begin{array}{l}
z_{n}=J_{r_{n}} x_{n}, \\
u_{n}=W_{r_{n}} x_{n}, \\
y_{n}=J^{-1}\left(\alpha_{n} J x_{n}+\beta_{n} J S^{n} z_{n}+\gamma_{n} J T^{n} u_{n}\right), \\
C_{n+1}=\left\{v \in C_{n}: \phi\left(v, y_{n}\right) \leq \phi\left(v, x_{n}\right)+\zeta_{n}\right\}, \\
x_{n+1}=\Pi_{C_{n+1}} x_{1}, \quad n \in \mathbb{N},
\end{array}\right.
$$

where $\left\{\alpha_{n}\right\},\left\{\beta_{n}\right\}$, and $\left\{\gamma_{n}\right\}$ are sequences in $(0,1)$ such that $\alpha_{n}+\beta_{n}+\gamma_{n}=1,\left\{r_{n}\right\} \subset[d, \infty)$ for some $d>0 \mu_{n}=\sup \left\{\mu_{n}^{S}, \mu_{n}^{T}\right\}, v_{n}=\sup \left\{v_{n}^{S}, v_{n}^{T}\right\}, \psi=\sup \left\{\psi^{S}, \psi^{T}\right\}$ for all $n \geq 1, \zeta=$ $v_{n} \sup _{q \in \mathcal{F}} \psi\left(\phi\left(q, x_{n}\right)\right)+\mu_{n}$. If $\lim _{n \rightarrow \infty} \alpha_{n} \beta_{n}=0$ and $\liminf _{n \rightarrow \infty} \alpha_{n} \gamma_{n}<1$, then $\left\{x_{n}\right\}$ converges strongly to $\Pi_{F} x_{1}$.

\section{Competing interests \\ The authors declare that they have no competing interests.}

Authors' contributions

All authors contributed equally and significantly in writing this article. All authors read and approved the final manuscript.

\section{Acknowledgements}

The authors would like to express their thanks to the reviewer for helpful suggestions and comments for the improvement of this paper. This work was supported by Thaksin University.

Received: 10 September 2012 Accepted: 27 December 2012 Published: 16 January 2013

\section{References}

1. Martinet, B: Regularization d'inéquations variationelles par approximations successives. Rev. Fr. Inform. Rech. Oper. 4 , 154-159 (1970)

2. Rockafellar, RT: Monotone operators and the proximal point algorithm. SIAM J. Control Optim. 14, 877-898 (1976)

3. Solodov, MV, Svaiter, BF: Forcing strong convergence of proximal point iterations in a Hilbert space. Math. Program., Ser. A 87, 189-202 (2000) 31

4. Cho, YJ, Kang, SM, Zhou, H: Approximate proximal point algorithms for finding zeroes of maximal monotone operators in Hilbert spaces. J. Inequal. Appl. 2008, Article ID 598191 (2008)

5. Wei, L, Cho, YJ: Iterative schemes for zero points of maximal monotone operators and fixed points of nonexpansive mappings and their applications. Fixed Point Theory Appl. 2008, Article ID 168468 (2008)

6. Qin, X, Cho, YJ, Kang, SM: Approximating zeros of monotone operators by proximal point algorithms. J. Glob. Optim. 46, 75-87 (2010) 
7. Song, Y, Kang, Jl, Cho, YJ: On iterations methods for zeros of accretive operators in Banach spaces. Appl. Math. Comput. 216, 1007-1017 (2010)

8. Cholamjiak, $\mathrm{P}, \mathrm{Cho}, \mathrm{YJ}$, Suantai, S: Composite iterative schemes for maximal monotone operators in reflexive Banach spaces. Fixed Point Theory Appl. 2011, Article ID 7 (2011)

9. Agarwal, RP, Zhou, HY, Cho, YJ, Kang, SM: Zeros and mappings theorems for perturbations of $m$-accretive operators in Banach spaces. Comput. Math. Appl. 49, 147-155 (2005)

10. Takahashi, W, Zembayashi, $\mathrm{K}:$ Strong convergence theorem by a new hybrid method for equilibrium problems and relatively nonexpansive mappings. Fixed Point Theory Appl. 2008, Article ID 528476 (2008)

11. Takahashi, W, Zembayashi, K: Strong and weak convergence theorems for equilibrium problems and relatively nonexpansive mappings in Banach spaces. Nonlinear Anal. 70, 45-57 (2009)

12. Reich, S: A weak convergence theorem for the alternating method with Bregman distances. In: Kartsatos, AG (ed.) Theory and Applications of Nonlinear Operators of Accretive and Monotone Type, pp. 313-318. Dekker, New York (1996)

13. Qin, X, Cho, YJ, Kang, SM: Convergence theorems of common elements for equilibrium problems and fixed point problems in Banach spaces. J. Comput. Appl. Math. 225, 20-30 (2009)

14. Zhou, H, Gao, G, Tan, B: Convergence theorems of a modified hybrid algorithm for a family of quasi- $\phi$-asymptotically nonexpansive mappings. J. Appl. Math. Comput. 32(2), 453-464 (2010)

15. Alber, Yal: Metric and generalized projection operators in Banach spaces: properties and applications. In: Kartsatos, A (ed.) Theory and Applications of Nonlinear Operators of Accretive and Monotone Type, pp. 15-50. Dekker, New York (1996)

16. Matsushita, S, Takahashi, W: A strong convergence theorem for relatively nonexpansive mappings in a Banach space. J. Approx. Theory 134, 257-266 (2005)

17. Boonchari, D, Saejung, S: Approximation of common fixed points of a countable family of relatively nonexpansive mappings. Fixed Point Theory Appl. 2010, Article ID 407651 (2010)

18. Aoyama, K, Kimura, Y, Takahashi, W, Toyoda, M: Approximation of common fixed points of a countable family of nonexpansive mappings in a Banach space. Nonlinear Anal. 67, 2350-2360 (2007)

19. Saewan, S, Kumam, P: A hybrid iterative scheme for a maximal monotone operator and two countable families of relatively quasi-nonexpansive mappings for generalized mixed equilibrium and variational inequality problems. Abstr. Appl. Anal. 2010, Article ID 123027 (2010)

20. Qin, X, Cho, SY, Kang, SM: On hybrid projection methods for asymptotically quasi- $\phi$-nonexpansive mappings. Appl. Math. Comput. 215, 3874-3883 (2010)

21. Alber, Y, Espínola, R, Lorenzo, P: Strong convergent approximations to fixed points of total asymptotically nonexpansive mappings. Acta Math. Sin. Engl. Ser. 24, 1005-1022 (2008)

22. Chang, S-S, Joseph Lee, HW, Chan, CK, Yang, L: Approximation theorems for total quasi- $\phi$-asymptotically nonexpansive mappings with applications. Appl. Math. Comput. 218, 2921-2931 (2011)

23. Chang, S-S, Joseph Lee, HW, Chan, CK, Yang, L: A modified Halpern-type iteration algorithm for totally quasi- $\phi$-asymptotically nonexpansive mappings with applications. Appl. Math. Comput. 218, 6489-6497 (2012)

24. Cioranescu, I: Geometry of Banach Spaces, Duality Mappings and Nonlinear Problems. Kluwer Academic, Dordrecht (1990)

25. Takahashi, W: Nonlinear Functional Analysis, Yokohama-Publishers, Yokohama (2000)

26. Reich, S: Geometry of Banach spaces, duality mappings and nonlinear problems. Bull. Am. Math. Soc. 26, 367-370 (1992)

27. Hudzik, H, Kowalewski, W, Lewicki, G: Approximative compactness and full rotundity in Musielak-Orlicz spaces and Lorentz-Orlicz spaces. Z. Anal. Anwend. 25, 163-192 (2006)

28. Kamimura, S, Takahashi, W: Strong convergence of a proximal-type algorithm in a Banach space. SIAM J. Optim. 13, 938-945 (2002)

29. Kohsaka, F, Takahashi, W: Existence and approximation of fixed points of firmly nonexpansive-type mappings in Banach spaces. SIAM J. Control Optim. 19(2), 824-835 (2008)

30. Cho, YJ, Zhou, HY, Guo, G: Weak and strong convergence theorems for three-step iterations with errors for asymptotically nonexpansive mappings. Comput. Math. Appl. 47, 707-717 (2004)

31. Rockafellar, RT: On the maximality of sums of nonlinear monotone operators. Trans. Am. Math. Soc. 149, 75-88 (1970)

32. Zegeye, $\mathrm{H}$, Shahzad, $\mathrm{N}$ : A hybrid scheme for finite families of equilibrium, variational inequality and fixed point problems. Nonlinear Anal. 74, 263-272 (2011)

doi:10.1186/1029-242X-2013-23

Cite this article as: Saewan and Kumam: A new iteration process for equilibrium, variational inequality, fixed point problems, and zeros of maximal monotone operators in a Banach space. Journal of Inequalities and Applications 2013 2013:23. 